\title{
Demographic and lifestyle factors associated with adherence to the Mediterranean diet in relation to overweight/obesity among Israeli adolescents: findings from the Mabat Israeli national youth health and nutrition survey
}

\author{
Wen Peng ${ }^{1,2}$, Rebecca Goldsmith ${ }^{3}$ and Elliot M Berry ${ }^{4, *}$ \\ 'Braun School of Public Health, Hebrew University - Hadassah Medical School, Jerusalem, Israel: ${ }^{2}$ Endocrinology \\ Department, Affiliated People's Hospital of Jiangsu University, Zhenjiang, People's Republic of China: ${ }^{3}$ Nutrition \\ Department, Public Health Services, Ministry of Health, Jerusalem, Israel: ${ }^{4}$ Department of Human Nutrition and \\ Metabolism, Braun School of Public Health, Hebrew University - Hadassah Medical School, 9112102 \\ Jerusalem, Israel
}

Submitted 9 August 2016: Accepted 14 September 2016: First published online 10 November 2016

\begin{abstract}
Objective: To investigate demographic and lifestyle factors associated with adherence to the Mediterranean diet (MD) in Israeli adolescents.

Design: Cross-sectional.

Setting: School-based.

Subjects: Schoolchildren ( $n$ 5268) aged 11-19 years answered self-administered questionnaires on food consumption, eating habits and lifestyle; a subset ( $n$ 578) also completed $24 \mathrm{~h}$ food recalls.

Results: Using a modified KIDMED index, $25 \cdot 5 \%$ of the students had poor, $55.2 \%$ had average and $19.3 \%$ had good MD adherence. Jewish middle-school children had the highest proportion (28.2\%) of poor MD adherence. Olive oil usage, derived from $24 \mathrm{~h}$ food recalls, was $18.1 \%$ in Jewish families $v .71 \cdot 1 \%$ in Arab homes. In Jewish boys, the odds (OR; $95 \% \mathrm{CI}$ ) of having poor MD adherence was higher in those who watched television/videos/listened to music for $\geq 2 \mathrm{~h} / \mathrm{d}(1 \cdot 25$; $0.98,1.58)$ and those who sometimes/don't read food labels $(1.69 ; 1.31,2 \cdot 18)$. In Jewish girls, the odds for having poor MD adherence was significantly higher in those whose mother's schooling was $<12$ years $(2 \cdot 06 ; 1 \cdot 41,3 \cdot 00)$ and those who sometimes/don't read food labels (1.35; 1.08, 1.69). In Arab boys, watching television/videos/listening to music for $\geq 2 \mathrm{~h} / \mathrm{d}$ was significantly associated with poor MD adherence $(1 \cdot 89 ; 1 \cdot 16,3 \cdot 07)$. In Arab girls, no aerobic activity or ball games weekly was associated with poor MD adherence (1.38; 0.91, 2.09).

Conclusions: Israeli adolescents had overall a high rate of poor MD adherence. Jewish middle-school children were at the highest risk. Interventions aimed at increasing physical activity, reducing sedentary time, improving mother's education and promoting reading of food labels are recommended.
\end{abstract}

Keywords

Mediterranean diet

KIDMED

Israeli adolescents

Overweight/obesity

MABAT
Childhood and adolescent overweight and obesity are major public health challenges in both their magnitude and consequences. In the USA, data in 2012 showed that more than one-third of children and adolescents were overweight and $18 \%$ were obese ${ }^{(1)}$. In Israeli adolescents, the prevalence was slightly lower, with $13-15 \%$ being overweight and 4-9\% being obese depending on gender and ethnicity ${ }^{(2)}$. Overweight and obesity in childhood and adolescence have significant negative impacts on health, which last long into adulthood. Studies have shown that adolescent obesity is associated with increased risk of end-stage renal failure ${ }^{(3)}, \mathrm{CVD}^{(4,5)}$ and some types of cancer $^{(5,6)}$. A recent Israeli follow-up study has shown that overweight and obesity in adolescence are strongly associated with increased cardiovascular mortality in adulthood $^{(7)}$.

Lifestyle including dietary change is one of the main causes of overweight and obesity. Among various dietary patterns, the Mediterranean diet (MD) has been accepted as one of the healthiest dietary models in the world. The MD has shown its health benefits in adults by reducing CVD, type 2 diabetes, certain cancers and some neurodegenerative diseases ${ }^{(8,9)}$. Meanwhile, the MD is also a common heritage of the culture and tradition in countries 
around the Mediterranean basin. In 2013, the MD was inscribed as an Intangible Cultural Heritage of Humanity by the United Nations Educational, Scientific and Cultural Organization $^{(10)}$. Nevertheless, against the background of global Westernization and urbanization, a trend of erosion in adherence to the MD is occurring, especially among children and adolescents ${ }^{(11)}$.

On the other hand, the characteristics of eating behaviour in adolescents also affect their dietary patterns. Adolescence is a critical time for the development of eating habits, which are a complex set of behaviours influenced by physiological, psychological, social and genetic factors ${ }^{(12)}$. Disordered eating, as one of the most common chronic conditions in adolescents ${ }^{(13)}$, influences adolescents' eating behaviours, thus affecting their diet. Data from an Israeli national survey showed that some $30 \%$ of Israeli girls met the criteria of disordered eating using a modified SCOFF questionnaire ${ }^{(14)}$.

Given the high prevalence of overweight/obesity and disordered eating in children and adolescents, as well as the healthy and cultural implications of the MD, it is important to investigate the dietary patterns in adolescents. This is to clarify the dietary features in this key population, identify the high-risk groups and suggest possible interventions for improvement. Since the Mediterranean Diet Quality Index (KIDMED), a tool to evaluate adherence to the MD in children and adolescents, was developed in 2004, it has been widely used in many Mediterranean basin countries, including Spain ${ }^{(11,15,16)}$, Italy ${ }^{(17,18)}$, Greece ${ }^{(19-22)}$, Turkey $^{(23)}$ and Cyprus ${ }^{(24)}$. However, adherence to the MD among Israeli adolescents has not yet been described.

To rectify this, the current paper describes the factors associated with adherence to the MD in Israeli adolescents based on an Israeli national youth health and nutrition survey (Mabat Youth Survey, 2003-2004). The data also allow study of possible associations between MD adherence and body weight.

\section{Methods}

\section{Population and sampling}

The study population, study design and sampling have been described in detail elsewhere ${ }^{(2)}$. Briefly, 6274 schoolchildren in grades 7 to 12 , aged 11-19 years, were enrolled into a cross-sectional, nationally representative, school-based study: the Mabat Youth Survey. Selfadministered questionnaires including FFQ, eating habits and lifestyle questionnaires were completed by 6274 adolescents, among whom 5268 finished the FFQ. The compliance rate for completion of the FFQ in Jewish and Arab participants was $87 \cdot 7$ and $76 \cdot 1 \%$, respectively. Adolescents who were in middle school, who were from low socio-economic status (SES) backgrounds or who did not perform aerobic activity or ball games weekly, had a higher rate of not finishing the FFQ. Five hundred and seventy-eight of the 5268 adolescents who completed an FFQ were also interviewed using the $24 \mathrm{~h}$ food recall method. Because the FFQ contained the majority of information on the dietary patterns, only the 5268 adolescents who completed the FFQ were included in the present KIDMED study. The original questionnaire was developed in Hebrew and then translated into Arabic. Data were collected in both Jewish and Arab, religious and non-religious schools. Schools from the Haredi (ultra orthodox) sector or boarding schools were not included.

\section{Data resources}

Adherence to the MD in adolescents was assessed using a modified KIDMED index ${ }^{(11)}$. The KIDMED index components were extracted from the self-administered FFQ or eating habits questionnaires. Other data related to the KIDMED index, which were not asked about specifically in the FFQ or eating habits questionnaires, were derived from the $24 \mathrm{~h}$ food recalls. Ethnicity (Jews or Arabs) was defined by the location of the school and the language of questionnaires used in the school. SES was taken from the Ministry of Education's classification of the welfare level of schools based primarily on the socio-economic level of the location. Other demographic and lifestyle factors were determined from answers to the self-administered questionnaires. Anthropometric indicators were measured by trained personnel.

\section{Composite variables}

\section{KIDMED score}

The modified KIDMED index used in the present study was composed of fourteen items adapted from the original sixteen-item KIDMED index. Items on olive oil usage at home and nut consumption (Table 1, items 15 and 16) were not included, because neither was specifically asked about in the FFQ of the Mabat Youth Survey. Olive oil usage at home was derived from the 578 adolescents who finished an additional $24 \mathrm{~h}$ food recall, but nut consumption was not available. Calculation of the modified fourteen-item KIDMED score was applied as shown in Table 1. In the original KIDMED index, three items (items 9, 11 and 12 in Table 1) specifically referred to consumption of designated food categories at breakfast. However, since the FFQ contained no information on meal times, daily consumption of designated food categories was defined as positive in the modified KIDMED index. From the $24 \mathrm{~h}$ food recalls, it was found that dairy products (item 11) and commercially baked goods or pastries (item 12) were not typically consumed at breakfast by Israeli schoolchildren. Cereals or grains (item 9) were a major component of breakfast, but were also eaten throughout the day.

The original KIDMED score was classified into three categories: (i) $\geq 8$, good; (ii) $4-7$, average; and (iii) $\leq 3$, poor. Based on the fraction of the upper limit to the score range, and the fact that two +1 points were lost in the 
Table 1 Definition of the modified KIDMED index used in the Mabat Youth Survey (2003-2004)

\begin{tabular}{|c|c|c|c|}
\hline No. & Score & Item & Comment \\
\hline 1 & +1 & Fruit or fruit juice daily & From FFQ \\
\hline 2 & +1 & Second fruit daily & From FFQ \\
\hline 3 & +1 & Fresh or cooked vegetables daily & From FFQ. Potatoes, pickles or vegetable soup were not included \\
\hline 4 & +1 & Fresh or cooked vegetables $>1$ time/d & From FFQ. Potatoes, pickles or vegetable soup were not included \\
\hline 5 & +1 & Regular fish consumption (at least 2-3 times/week) & From FFQ \\
\hline 6 & -1 & Fast-food restaurant $>1$ time/week & $\begin{array}{l}\text { From question in eating habits questionnaire on times of monthly eating } \\
\text { fast food (falafel, hamburger, pizza). Answer with }>4 \text { times/month was } \\
\text { defined as positive }\end{array}$ \\
\hline 7 & +1 & Pulses $>1$ time/week & From FFQ \\
\hline 8 & +1 & Pasta or rice almost daily ( $\geq 5$ times/week) & From FFQ \\
\hline 9 & +1 & Cereals or grains (bread, etc.) for breakfast & $\begin{array}{l}\text { From FFQ. Daily consumption was defined as positive, without } \\
\text { specifically referring to breakfast }\end{array}$ \\
\hline 10 & -1 & No breakfast & $\begin{array}{l}\text { From questionnaire on eating habits. Answer with 'didn't eat breakfast } \\
\text { yesterday' was defined as positive }\end{array}$ \\
\hline 11 & +1 & A dairy product for breakfast (yoghurt, milk, etc.) & $\begin{array}{l}\text { From FFQ. Daily consumption was defined as positive, without } \\
\text { specifically referring to breakfast }\end{array}$ \\
\hline 12 & -1 & Commercially baked goods or pastries for breakfast & $\begin{array}{l}\text { From FFQ. Daily consumption was defined as positive, without } \\
\text { specifically referring to breakfast }\end{array}$ \\
\hline 13 & +1 & Two yoghurts and/or some cheese $(40 \mathrm{~g})$ daily & From FFQ. One yoghurt and $20 \mathrm{~g}$ cheese were also defined as positive \\
\hline 14 & -1 & Sweets and candy several times daily & From FFQ. Several was defined as $\geq 3$ \\
\hline 15 & +1 & Olive oil usage at home & $\begin{array}{l}\text { No information in FFQ. Extracted from } 24 \mathrm{~h} \text { food recalls. Not included in } \\
\text { the modified KIDMED score }\end{array}$ \\
\hline 16 & +1 & Regular nuts consumption (at least $2-3$ times/week) & $\begin{array}{l}\text { No specific information in Mabat Youth Survey. Not included in the } \\
\text { modified KIDMED score }\end{array}$ \\
\hline
\end{tabular}

KIDMED, Mediterranean Diet Quality Index.

fourteen-item KIDMED index, we defined cut-off points for the modified fourteen-item KIDMED index as follows: (i) $\geq 7$, good; (ii) 3-6, average; and (iii) $\leq 2$, poor.

\section{$B M I$}

BMI was categorized based on the age- and sex-specific cut-off values of the Centers for Disease Control and Prevention/National Center for Health Statistics 2000 growth charts. Four categories were defined as underweight ( $<5$ th percentile), normal weight ( 5 th to 85 th percentile), overweight (85th to 95th percentile) and obese ( $>95$ th percentile), which was previously described elsewhere $^{(2)}$.

\section{Disordered eating}

Disordered eating was assessed using an adapted fouritem SCOFF questionnaire compared with the original five-item SCOFF questionnaire, which was used to screen for eating disorders ${ }^{(25)}$. The question on weight loss was modified from original $6.35 \mathrm{~kg}$ (1 stone) in three months to $3 \mathrm{~kg}$ in our questionnaire ${ }^{(14)}$, in order to adapt to the general lower body weight of adolescents as compared with adults. The cut-off point for the four-item screening test was the same as the original one; that is, two or more affirmative answers in the questionnaire was categorized as disordered eating (positive) ${ }^{(14)}$.

\section{Statistical analyses}

All statistical analyses were performed using the statistical software package IBM SPSS Statistics Version 20.0. The $\chi^{2}$ test was used to compare the distribution of KIDMED scores. Student's $t$ test or one-way ANOVA was used to compare the mean of KIDMED scores. When one-way
ANOVA showed significant difference among groups, the Bonferroni correction was used to account for the inflation in Type I error in the multiple comparisons. Adjusted odds ratios were calculated by binary logistic regression. A $P$ value of $<0.05$ was considered as significant.

\section{Results}

Among the 5268 schoolchildren between 11 and 19 years old, $43.5 \%$ ( $n$ 2290) were boys and 56.5\% ( $n$ 2978) were girls; $50 \cdot 6 \%$ ( $n$ 2665) were in middle school and $49 \cdot 4 \%$ ( $n$ 2603) were in high school; 74.7\% ( $n$ 3936) were Jews and $25.3 \%$ ( $n$ 1332) were Arabs.

Among the Jewish children, $20 \cdot 3 \%$ ( $n$ 322) of the boys and $15.7 \%$ ( $n$ 306) of the girls were overweight or obese; among the Arab children, $23 \cdot 1 \%$ ( $n$ 122) of the boys and $21.7 \%$ ( $n$ 159) of the girls were overweight or obese.

\section{KIDMED score by demographic and lifestyle factors}

Table 2 shows the distribution of the modified fourteen-item KIDMED scores by school level, gender and ethnicity. Items 1-4 showed that 20-30\% more Arab adolescents than Jewish adolescents consumed fruits and vegetables daily. By contrast, Arabs ate fewer dairy products (items 11 and 13) and more sweets and candies (item 14) than Jews. Regarding eating habits, Arabs consumed more fast foods (as shown in item 6), with the highest rate (73.5\%) in Arab boys in high school, which was more than $30 \%$ higher than that in Jewish boys of the same age group (41.7\%). Girls generally had a $15-20 \%$ higher rate of skipping breakfast than boys, with Jewish girls in high school skipping breakfast the 
Table 2 Distribution of the modified fourteen-item KIDMED index in Israeli adolescents aged 11-19 years by school level, gender and ethnicity; Mabat Youth Survey (2003-2004)

\begin{tabular}{|c|c|c|c|c|c|c|c|c|c|c|c|c|c|}
\hline & & \multicolumn{6}{|c|}{ Middle school (n 2665) } & \multicolumn{6}{|c|}{ High school (n 2603) } \\
\hline & & \multicolumn{2}{|c|}{ Boys } & \multicolumn{2}{|c|}{ Girls } & \multicolumn{2}{|c|}{ Total } & \multicolumn{2}{|c|}{ Boys } & \multicolumn{2}{|c|}{ Girls } & \multicolumn{2}{|c|}{ Total } \\
\hline & & $n$ & $\%$ & $n$ & $\%$ & $n$ & $\%$ & $n$ & $\%$ & $n$ & $\%$ & $n$ & $\%$ \\
\hline \multirow[t]{3}{*}{1} & \multicolumn{13}{|c|}{ Fruit or fruit juice daily } \\
\hline & Jews & 453 & 47.0 & 566 & $51 \cdot 1$ & 1019 & $49 \cdot 2$ & 395 & 51.5 & 574 & $52 \cdot 2$ & 969 & 51.9 \\
\hline & Arabs & 208 & 73.2 & 256 & $82 \cdot 3$ & 464 & 78.0 & 195 & $70 \cdot 7$ & 326 & $70 \cdot 7$ & 521 & $70 \cdot 7$ \\
\hline \multirow[t]{3}{*}{2} & \multicolumn{13}{|c|}{ Second fruit daily } \\
\hline & Jews & 346 & 35.9 & 417 & $37 \cdot 7$ & 763 & $36 \cdot 9$ & 298 & 38.9 & 438 & 39.9 & 736 & 39.4 \\
\hline & Arabs & 186 & 65.5 & 218 & $70 \cdot 1$ & 404 & 67.9 & 165 & $59 \cdot 8$ & 271 & $58 \cdot 8$ & 436 & $59 \cdot 2$ \\
\hline \multirow[t]{3}{*}{3} & \multicolumn{13}{|c|}{ Fresh or cooked vegetables daily } \\
\hline & Jews & 419 & 43.5 & 579 & $52 \cdot 3$ & 998 & $48 \cdot 2$ & 370 & $48 \cdot 2$ & 626 & $57 \cdot 0$ & 996 & $53 \cdot 4$ \\
\hline & Arabs & 207 & 72.9 & 232 & 74.6 & 439 & 73.8 & 217 & 78.6 & 335 & $72 \cdot 7$ & 552 & 74.9 \\
\hline \multirow[t]{3}{*}{4} & \multicolumn{13}{|c|}{ Fresh or cooked vegetables $>1$ time/d } \\
\hline & Jews & 340 & $35 \cdot 3$ & 467 & $42 \cdot 2$ & 807 & $39 \cdot 0$ & 308 & $40 \cdot 2$ & 540 & $49 \cdot 1$ & 848 & $45 \cdot 4$ \\
\hline & Arabs & 184 & $64 \cdot 8$ & 205 & 65.9 & 389 & 65.4 & 190 & $68 \cdot 8$ & 299 & 64.9 & 489 & $66 \cdot 4$ \\
\hline \multirow[t]{3}{*}{5} & \multicolumn{13}{|c|}{ Regular fish consumption (at least $2-3$ times/week) } \\
\hline & Jews & 378 & $39 \cdot 3$ & 445 & $40 \cdot 2$ & 823 & $39 \cdot 8$ & 350 & $45 \cdot 6$ & 417 & 37.9 & 767 & $41 \cdot 1$ \\
\hline & Arabs & 121 & $42 \cdot 6$ & 121 & 38.9 & 242 & $40 \cdot 7$ & 89 & $32 \cdot 2$ & 133 & 28.9 & 222 & $30 \cdot 1$ \\
\hline \multirow[t]{3}{*}{6} & \multicolumn{13}{|c|}{ Fast-food restaurant $>1$ time/week } \\
\hline & Jews & 351 & $37 \cdot 7$ & 289 & $27 \cdot 0$ & 640 & $32 \cdot 0$ & 312 & 41.7 & 299 & $28 \cdot 0$ & 611 & 33.6 \\
\hline & Arabs & 143 & $55 \cdot 9$ & 130 & $43 \cdot 6$ & 273 & $49 \cdot 3$ & 194 & 73.5 & 212 & $47 \cdot 1$ & 406 & $56 \cdot 9$ \\
\hline 7 & Pulses > & $\mathrm{me} / \mathrm{we}$ & & & & & & & & & & & \\
\hline & Jews & 809 & $84 \cdot 0$ & 939 & $84 \cdot 8$ & 1748 & 84.4 & 682 & 88.9 & 947 & $86 \cdot 2$ & 1629 & $87 \cdot 3$ \\
\hline & Arabs & 269 & $94 \cdot 7$ & 284 & $91 \cdot 3$ & 553 & $92 \cdot 9$ & 261 & $94 \cdot 6$ & 409 & $88 \cdot 7$ & 670 & $90 \cdot 9$ \\
\hline 8 & Pasta or & almo & Jaily $(\geq 5$ & nes/w & & & & & & & & & \\
\hline & Jews & 486 & 50.5 & 544 & 49.1 & 1030 & $49 \cdot 8$ & 391 & $51 \cdot 0$ & 506 & $46 \cdot 0$ & 897 & $48 \cdot 1$ \\
\hline & Arabs & 126 & 44.4 & 147 & $47 \cdot 3$ & 273 & $45 \cdot 9$ & 103 & 37.3 & 161 & 34.9 & 264 & 35.8 \\
\hline 9 & Cereal or & real $p$ & uct daily & & & & & & & & & & \\
\hline & Jews & 686 & 71.2 & 737 & $66 \cdot 6$ & 1423 & $68 \cdot 7$ & 573 & 74.7 & 774 & 70.4 & 1347 & $72 \cdot 2$ \\
\hline & Arabs & 233 & $82 \cdot 0$ & 256 & $82 \cdot 3$ & 489 & $82 \cdot 2$ & 247 & 89.5 & 356 & $77 \cdot 2$ & 603 & $81 \cdot 8$ \\
\hline 10 & No break & & & & & & & & & & & & \\
\hline & Jews & 226 & 24.0 & 443 & $40 \cdot 8$ & 669 & 33.0 & 195 & $25 \cdot 8$ & 480 & 44.5 & 675 & $36 \cdot 8$ \\
\hline & Arabs & 31 & 11.0 & 90 & 29.0 & 121 & 20.5 & 67 & $25 \cdot 1$ & 185 & 41.0 & 252 & $35 \cdot 1$ \\
\hline 11 & Dairy pro & t daily & & & & & & & & & & & \\
\hline & Jews & 707 & 73.4 & 754 & $68 \cdot 1$ & 1461 & $70 \cdot 6$ & 545 & 71.7 & 686 & 62.4 & 1231 & $66 \cdot 0$ \\
\hline & Arabs & 173 & 60.9 & 161 & 51.8 & 334 & $56 \cdot 1$ & 155 & $56 \cdot 2$ & 222 & 48.2 & 377 & $51 \cdot 2$ \\
\hline 12 & Commerc & y bak & goods 0 & astries & & & & & & & & & \\
\hline & Jews & 356 & 37.0 & 404 & 36.5 & 760 & $36 \cdot 7$ & 286 & $37 \cdot 3$ & 403 & $36 \cdot 7$ & 689 & 36.9 \\
\hline & Arabs & 116 & $40 \cdot 8$ & 165 & $53 \cdot 1$ & 281 & $47 \cdot .2$ & 118 & $42 \cdot 8$ & 170 & 36.9 & 288 & $39 \cdot 1$ \\
\hline 13 & Two yogh & $\mathrm{s}$ and & $40 \mathrm{~g}$ che & e daily & & & & & & & & & \\
\hline & Jews & 409 & 42.5 & 412 & $37 \cdot 2$ & 821 & 39.7 & 332 & 43.3 & 381 & 34.7 & 713 & 38.2 \\
\hline & Arabs & 83 & $29 \cdot 2$ & 72 & $23 \cdot 2$ & 155 & $26 \cdot 1$ & 74 & $26 \cdot 8$ & 76 & $16 \cdot 5$ & 150 & $20 \cdot 4$ \\
\hline 14 & Sweets a & candy & veral tin & daily & & & & & & & & & \\
\hline & Jews & 94 & $9 \cdot 8$ & 143 & 12.9 & 237 & 11.4 & 81 & $10 \cdot 6$ & 113 & $10 \cdot 3$ & 194 & $10 \cdot 4$ \\
\hline & Arabs & 73 & $25 \cdot 7$ & 93 & 29.9 & 166 & $27 \cdot 9$ & 73 & $26 \cdot 4$ & 119 & $25 \cdot 8$ & 192 & $26 \cdot 1$ \\
\hline
\end{tabular}

KIDMED, Mediterranean Diet Quality Index.

Among 5268 participants, 181 and 100 responses were missing on items 6 and 10, respectively.

most (44.5\%; item 10). Besides the fourteen items in Table 1, olive oil usage at home was summarized from 578 adolescents from their $24 \mathrm{~h}$ food recalls, which showed that $18.1 \%$ (80/443) of Jewish and $71 \cdot 1 \%(96 / 135)$ of Arab families used olive oil at home.

Table 3 shows the categorization of the fourteen-item KIDMED scores. Of the total population, $25.5 \%$ ( $n$ 1276) had poor, $55 \cdot 2 \%$ ( $n$ 2761) had average and 19.3\% ( $n$ 968) had good KIDMED scores. Further analysis by school level, gender and ethnicity showed that Jewish middleschool children had the highest proportion (28.0\% in boys and $28.4 \%$ in girls) with a poor KIDMED score, while Arab middle-school children had the highest proportion (boys $26.9 \%$ and girls $23.2 \%$ ) with a good KIDMED score.
Table 4 presents the comparison of KIDMED scores stratified by demographic features and selected lifestyle factors. In Jews, high-school children had higher KIDMED scores than middle-school children, and the difference was close to significant in Jewish boys $(P=0.053)$. In contrast, in Arabs, middle-school children had significantly higher KIDMED scores than high-school children (Arab boys $P=0.023$; Arab girls $P<0 \cdot 001$ ). No significant difference was observed in KIDMED scores between groups according to SES. Mother's school education ( $\geq 12$ years) was positively associated with higher KIDMED scores in all the groups, with a significant difference in Jewish boys $(P=0 \cdot 012)$ and Jewish girls $(P=0 \cdot 001)$. Both Jewish boys and Arab boys who smoked had lower KIDMED scores 
Table 3 Categorization of the modified fourteen-item KIDMED scores in Israeli adolescents aged 11-19 years by school level, gender and ethnicity; Mabat Youth Survey (2003-2004)

\begin{tabular}{|c|c|c|c|c|c|c|c|c|c|c|c|c|c|c|}
\hline \multirow[b]{3}{*}{ KIDMED category } & \multicolumn{7}{|c|}{ Middle school $(n 2519$, missing $=146)$} & \multicolumn{7}{|c|}{ High school $(n 2486$, missing $=117)$} \\
\hline & \multicolumn{2}{|c|}{ Boys } & \multicolumn{2}{|c|}{ Girls } & \multicolumn{2}{|c|}{ Total } & \multirow[b]{2}{*}{$P$ (boys $v$. girls) } & \multicolumn{2}{|c|}{ Boys } & \multicolumn{2}{|c|}{ Girls } & \multicolumn{2}{|c|}{ Total } & \multirow[b]{2}{*}{$P$ (boys $v$. girls) } \\
\hline & $n$ & $\%$ & $n$ & $\%$ & $n$ & $\%$ & & $n$ & $\%$ & $n$ & $\%$ & $n$ & $\%$ & \\
\hline \multicolumn{15}{|l|}{ Poor (score $\leq 2$ ) } \\
\hline Jews & 256 & 28.0 & 299 & $28 \cdot 4$ & 555 & $28 \cdot 2$ & 0.838 & 174 & 23.5 & 291 & $27 \cdot 7$ & 465 & $26 \cdot 0$ & 0.049 \\
\hline Arabs & 44 & $17 \cdot 4$ & 54 & $18 \cdot 1$ & 98 & $17 \cdot 8$ & 0.823 & 54 & $21 \cdot 2$ & 104 & $23 \cdot 6$ & 158 & $22 \cdot 7$ & 0.465 \\
\hline$P$ (Jews v. Arabs) & \multicolumn{2}{|c|}{$0.001^{* *}$} & \multicolumn{2}{|c|}{$<0.001^{\star *}$} & \multicolumn{2}{|c|}{$<0.001^{* *}$} & & \multicolumn{2}{|c|}{0.438} & \multicolumn{2}{|c|}{0.101} & \multicolumn{2}{|c|}{0.091} & \\
\hline \multicolumn{15}{|c|}{ Average $($ score $=3-6)$} \\
\hline Jews & 484 & $52 \cdot 9$ & 551 & $52 \cdot 3$ & 1035 & $52 \cdot 6$ & 0.801 & 422 & $57 \cdot 1$ & 562 & 53.5 & 984 & $55 \cdot 0$ & 0.128 \\
\hline Arabs & \multirow{2}{*}{\multicolumn{2}{|c|}{0.424}} & 175 & $58 \cdot 7$ & 316 & 57.4 & 0.479 & 151 & $59 \cdot 2$ & 275 & $62 \cdot 4$ & & $61 \cdot 2$ & 0.412 \\
\hline$P$ (Jews v. Arabs) & & & \multicolumn{2}{|c|}{0.051} & \multicolumn{2}{|c|}{$0.048^{\star}$} & & \multicolumn{2}{|c|}{0.556} & \multicolumn{2}{|c|}{$0.002^{\star \star}$} & \multicolumn{2}{|c|}{$0.005^{\star \star}$} & \\
\hline \multicolumn{15}{|l|}{ Good (score $\geq 7$ ) } \\
\hline Jews & 175 & $19 \cdot 1$ & 203 & $19 \cdot 3$ & 378 & $19 \cdot 2$ & 0.932 & 143 & $19 \cdot 4$ & 198 & $18 \cdot 8$ & 341 & $19 \cdot 1$ & 0.786 \\
\hline Arabs & 68 & 26.9 & 69 & $23 \cdot 2$ & 137 & 24.9 & 0.314 & 50 & $19 \cdot 6$ & 62 & $14 \cdot 1$ & 112 & $16 \cdot 1$ & 0.055 \\
\hline$P$ (Jews $v$ Arabs) & \multicolumn{2}{|c|}{$0.007^{\star \star}$} & \multicolumn{2}{|c|}{$0 \cdot 141$} & \multicolumn{2}{|c|}{$0.004^{\star \star}$} & & \multicolumn{2}{|c|}{0.929} & \multicolumn{2}{|c|}{$0.026^{\star}$} & \multicolumn{2}{|c|}{0.086} & \\
\hline
\end{tabular}

KIDMED, Mediterranean Diet Quality Index.

${ }^{*} P<0.05 .{ }^{* \star} P<0.01$.

Table 4 Modified fourteen-item KIDMED scores in Israeli adolescents aged 11-19 years by demographic features and lifestyle factors; Mabat Youth Survey (2003-2004)

\begin{tabular}{|c|c|c|c|c|c|c|c|c|c|c|c|c|c|c|c|c|}
\hline & \multicolumn{4}{|c|}{ Jewish boys } & \multicolumn{4}{|c|}{ Jewish girls } & \multicolumn{4}{|c|}{ Arab boys } & \multicolumn{4}{|c|}{ Arab girls } \\
\hline & $n$ & Mean & SD & $P$ & $n$ & Mean & SD & $P$ & $n$ & Mean & SD & $P$ & $n$ & Mean & SD & $P$ \\
\hline \multicolumn{17}{|l|}{ Grade } \\
\hline Middle school & 915 & $4 \cdot 14$ & $2 \cdot 39$ & \multirow[t]{2}{*}{0.053} & 1053 & $4 \cdot 14$ & $2 \cdot 49$ & \multirow[t]{2}{*}{0.832} & 253 & 4.92 & $2 \cdot 41$ & \multirow[t]{2}{*}{$0.023^{*}$} & 298 & 4.74 & $2 \cdot 28$ & \multirow[t]{2}{*}{$<0.001^{\star \star}$} \\
\hline High school & 739 & 4.36 & $2 \cdot 32$ & & 1051 & $4 \cdot 16$ & $2 \cdot 49$ & & 255 & 4.45 & $2 \cdot 19$ & & 441 & $4 \cdot 11$ & $2 \cdot 23$ & \\
\hline \multicolumn{17}{|c|}{ Socio-economic status } \\
\hline Low & 704 & 4.19 & $2 \cdot 36$ & \multirow{2}{*}{0.552} & 1042 & $4 \cdot 22$ & $2 \cdot 49$ & \multirow[t]{2}{*}{$0 \cdot 169$} & 266 & 4.77 & $2 \cdot 27$ & \multirow{2}{*}{0.309} & 374 & $4 \cdot 34$ & $2 \cdot 25$ & \multirow[t]{2}{*}{0.721} \\
\hline High & 950 & $4 \cdot 27$ & $2 \cdot 37$ & & 1062 & 4.07 & $2 \cdot 49$ & & 236 & 4.56 & $2 \cdot 38$ & & 355 & 4.40 & $2 \cdot 31$ & \\
\hline \multicolumn{17}{|l|}{ Mother's education } \\
\hline $\begin{array}{c}<12 \text { years of } \\
\text { schooling }\end{array}$ & 79 & $3 \cdot 67$ & $2 \cdot 10$ & \multirow[t]{2}{*}{$0.012^{*}$} & 135 & 3.54 & $2 \cdot 42$ & \multirow[t]{2}{*}{$0.001^{* *}$} & 146 & 4.45 & $2 \cdot 20$ & \multirow[t]{2}{*}{0.287} & 235 & $4 \cdot 13$ & $2 \cdot 16$ & \multirow[t]{2}{*}{$0 \cdot 139$} \\
\hline $\begin{array}{l}\geq 12 \text { years of } \\
\text { schooling }\end{array}$ & 1198 & $4 \cdot 37$ & $2 \cdot 40$ & & 1496 & $4 \cdot 31$ & $2 \cdot 48$ & & 278 & $4 \cdot 70$ & $2 \cdot 38$ & & 411 & 4.40 & $2 \cdot 31$ & \\
\hline \multicolumn{17}{|c|}{ Night sleeping time/d } \\
\hline$<6 \mathrm{~h}$ & 79 & 4.09 & $2 \cdot 40$ & \multirow[t]{2}{*}{0.489} & 90 & 3.97 & $2 \cdot 69$ & \multirow{2}{*}{0.450} & 14 & 4.50 & $1 . .95$ & \multirow{2}{*}{0.731} & 26 & 4.00 & $2 \cdot 58$ & \multirow[t]{2}{*}{0.427} \\
\hline$\geq 6 \mathrm{~h}$ & 1482 & $4 \cdot 28$ & $2 \cdot 37$ & & 1952 & $4 \cdot 17$ & $2 \cdot 48$ & & 464 & 4.72 & $2 \cdot 32$ & & 681 & 4.36 & $2 \cdot 27$ & \\
\hline Smoking (cigarett & okah) & & & & & & & & & & & & & & & \\
\hline Yes & 361 & 4.12 & $2 \cdot 33$ & $0 \cdot 198$ & 313 & $4 \cdot 11$ & $2 \cdot 60$ & 0.816 & 89 & $4 \cdot 20$ & $2 \cdot 35$ & $0.027^{\star}$ & 10 & 3.40 & $2 \cdot 17$ & 0.185 \\
\hline No & 1197 & $4 \cdot 30$ & $2 \cdot 37$ & & 1672 & $4 \cdot 14$ & $2 \cdot 47$ & & 401 & 4.81 & $2 \cdot 31$ & & 664 & $4 \cdot 35$ & $2 \cdot 26$ & \\
\hline Aerobic activity or & ames $\mathrm{w}$ & veekly & & & & & & & & & & & & & & \\
\hline Yes & 1348 & $4 \cdot 31$ & $2 \cdot 38$ & $0.009^{\star \star}$ & 1129 & $4 \cdot 24$ & 2.51 & 0.080 & 440 & 4.75 & $2 \cdot 28$ & 0.094 & 571 & 4.42 & $2 \cdot 26$ & 0.177 \\
\hline No & 306 & 3.92 & $2 \cdot 27$ & & 975 & 4.05 & $2 \cdot 47$ & & 68 & 4.25 & 2.51 & & 168 & $4 \cdot 15$ & $2 \cdot 31$ & \\
\hline Watch television/v & or lister & $\mathrm{n}$ to $\mathrm{mu}$ & usic & & & & & & & & & & & & & \\
\hline$\geq 2 \mathrm{~h} / \mathrm{d}$ & 684 & 4.07 & $2 \cdot 30$ & $0.010^{\star}$ & 1114 & 4.00 & $2 \cdot 48$ & $0.003^{\star \star}$ & 219 & $4 \cdot 30$ & $2 \cdot 34$ & $0.001^{\star *}$ & 445 & $4 \cdot 14$ & $2 \cdot 19$ & $0.001^{* \star}$ \\
\hline$<2 \mathrm{~h} / \mathrm{d}$ & 937 & $4 \cdot 37$ & $2 \cdot 40$ & & 958 & $4 \cdot 32$ & $2 \cdot 48$ & & 284 & 5.00 & $2 \cdot 23$ & & 284 & 4.70 & $2 \cdot 36$ & \\
\hline Dieting & & & & & & & & & & & & & & & & \\
\hline Yes & 243 & $4 \cdot 22$ & $2 \cdot 32$ & 0.886 & 681 & $4 \cdot 27$ & $2 \cdot 63$ & 0.105 & 70 & $5 \cdot 21$ & $2 \cdot 32$ & $0.039^{*}$ & 196 & $4 \cdot 27$ & $2 \cdot 18$ & 0.506 \\
\hline No & 1396 & $4 \cdot 25$ & $2 \cdot 37$ & & 1407 & 4.08 & $2 \cdot 42$ & & 436 & $4 \cdot 60$ & $2 \cdot 31$ & & 540 & 4.40 & $2 \cdot 30$ & \\
\hline Read food labels & & & & & & & & & & & & & & & & \\
\hline Always/often & 588 & 4.61 & $2 \cdot 32$ & $<0.001^{\star *}$ & 1000 & $4 \cdot 34$ & $2 \cdot 51$ & $<0.001^{\star \star}$ & 244 & 4.98 & $2 \cdot 30$ & $0.003^{\star \star}$ & 460 & 4.43 & $2 \cdot 22$ & 0.400 \\
\hline Sometimes/no & 1019 & 4.05 & $2 \cdot 37$ & & 1061 & 3.94 & $2 \cdot 46$ & & 251 & 4.37 & $2 \cdot 32$ & & 266 & 4.29 & $2 \cdot 34$ & \\
\hline Body weight & & & & & & & & & & & & & & & & \\
\hline Underweight & 90 & $3 \cdot 61$ & $2 \cdot 29$ & $0.021^{*}$ & 74 & 3.81 & $2 \cdot 16$ & 0.061 & 21 & 4.43 & $2 \cdot 80$ & 0.821 & 21 & $5 \cdot 14$ & 2.03 & 0.360 \\
\hline Normal weight & 1116 & 4.34 & 2.39 & & 1488 & $4 \cdot 16$ & 2.46 & & 349 & 4.71 & $2 \cdot 26$ & & 525 & 4.33 & $2 \cdot 32$ & \\
\hline Overweight & 201 & 4.09 & $2 \cdot 27$ & & 231 & $4 \cdot 12$ & $2 \cdot 56$ & & 63 & 4.52 & $2 \cdot 38$ & & 118 & 4.19 & $2 \cdot 19$ & \\
\hline Obese & 109 & 4.05 & $2 \cdot 51$ & & 64 & $4 \cdot 91$ & $2 \cdot 69$ & & 44 & $4 \cdot 89$ & 2.63 & & 36 & 4.50 & $2 \cdot 44$ & \\
\hline
\end{tabular}

KIDMED, Mediterranean Diet Quality Index.

${ }^{*} P<0.05,{ }^{*} P<0.01$.

than those who did not, and the difference was statistically significant in Arab boys $(P=0 \cdot 027)$. In all the groups, students who performed aerobic activity or ball games weekly had higher KIDMED scores than their comparison group, with significance in Jewish boys $(P=0 \cdot 009)$. Watching television/videos or listening to music for $\geq 2 \mathrm{~h} / \mathrm{d}$ 
was significantly associated with poorer KIDMED scores in all groups. Children who always/often read food labels consistently had better KIDMED scores than children who sometimes/don't read food labels, and the difference was significant in Jewish boys, Jewish girls and Arab boys. In Jewish boys, one-way ANOVA showed that body weight was associated with KIDMED score $(P=0.021)$; a further post hoc Bonferroni test revealed that the real difference existed between the underweight group and the normal weight group $(P=0 \cdot 032)$.

\section{Explanatory factors of KIDMED score}

Table 5 shows the adjusted odds ratios for having poor KIDMED scores by gender, ethnicity and selected lifestyle factors.

In Jewish boys, being in high school was negatively associated with having a poor KIDMED score $(\mathrm{OR}=0.72$; 95\% CI $0.57,0.92)$. Watching television/videos/listening to music for $\geq 2 \mathrm{~h} / \mathrm{d}$, sometimes/not reading food labels and being underweight were positively associated with having a poor KIDMED score, with statistical significance for sometimes/not reading food labels (OR $=1.69 ; 95 \%$ CI $1.31,2 \cdot 18)$ and being underweight $(\mathrm{OR}=1.88$; $95 \%$
CI 1.19, 2.98). Overweight and obesity were not explanatory factors for having a poor KIDMED score.

In Jewish girls, SES, mother's school education, sedentary lifestyle and reading food labels were associated with adherence to the MD. The odds of having a poor KIDMED score was significantly higher in groups who had higher SES (OR $=1.33$; $95 \%$ CI 1.06, 1.67), whose mother's years of education was $<12$ years $(\mathrm{OR}=2 \cdot 06$; $95 \% \mathrm{CI} 1 \cdot 41,3 \cdot 00)$ and who sometimes/don't read food labels $(\mathrm{OR}=1.35$; $95 \%$ CI 1.08, 1.69).

In Arab boys, watching television/videos/listening to music for $\geq 2 \mathrm{~h} / \mathrm{d}$ and sometimes/not reading food labels were associated with having a poor KIDMED score. The odds of having a poor KIDMED score if watching television/videos/listening to music for $\geq 2 \mathrm{~h} / \mathrm{d}$ was 1.89 times higher than that of the comparison group in Arab boys.

In Arab girls, no aerobic activity or ball games weekly or sometimes/not reading food labels were associated with a poor KIDMED score, but without statistical significance.

A further analysis into reading food labels showed that the proportion always/often reading food labels was significantly higher in girls than in boys $(52.4 v$. $39.6 \%, P<0 \cdot 001)$. A higher percentage of Arab children

Table 5 Adjusted odds ratios of having a poor KIDMED score ( $v$. average/good) among Israeli adolescents aged 11-19 years by gender, ethnicity and lifestyle factors; Mabat Youth Survey (2003-2004)

\begin{tabular}{|c|c|c|c|}
\hline & OR & $95 \% \mathrm{Cl}$ & $P$ \\
\hline \multicolumn{4}{|l|}{ Jewish boys $(n 1459$, missing $=271)$} \\
\hline \multicolumn{4}{|l|}{ School level } \\
\hline High/middle school & 0.72 & $0.57,0.92$ & $0.007^{\star \star}$ \\
\hline \multicolumn{4}{|l|}{ Watch television/videos/listen to music daily } \\
\hline$\geq 2 \mathrm{~h} /<2 \mathrm{~h}$ & 1.25 & $0.98,1.58$ & 0.073 \\
\hline \multicolumn{4}{|l|}{ Read food labels } \\
\hline Sometimes and no/always and often & 1.69 & $1 \cdot 31,2 \cdot 18$ & $<0.001^{\star \star}$ \\
\hline \multicolumn{4}{|l|}{ Body weight } \\
\hline Underweight/normal weight & 1.88 & $1 \cdot 19,2 \cdot 98$ & $0.007^{\star \star}$ \\
\hline Overweight/normal weight & 1.07 & $0.75,1.52$ & 0.709 \\
\hline Obese/normal weight & $1 \cdot 15$ & $0.72,1.84$ & 0.555 \\
\hline \multicolumn{4}{|l|}{ Jewish girls $(n 1588$, missing $=618)$} \\
\hline \multicolumn{4}{|l|}{ Socio-economic status } \\
\hline High/low & 1.33 & $1.06,1.67$ & $0.014^{*}$ \\
\hline \multicolumn{4}{|l|}{ Mother's education in school } \\
\hline$<12$ years $/ \geq 12$ years & 2.06 & $1.41,3.00$ & $<0.001^{\star *}$ \\
\hline \multicolumn{4}{|l|}{ Watch television/videos/listen to music daily } \\
\hline$\geq 2 \mathrm{~h} /<2 \mathrm{~h}$ & $1 \cdot 20$ & $0.95,1.50$ & 0.120 \\
\hline \multicolumn{4}{|l|}{ Read food labels } \\
\hline Sometimes and no/always and often & 1.35 & $1.08,1.69$ & $0.008^{* \star}$ \\
\hline \multicolumn{4}{|l|}{ Arab boys $(n 412$, missing $=148)$} \\
\hline \multicolumn{4}{|l|}{ Mother's education in school } \\
\hline$<12$ years $/ \geq 12$ year & 0.67 & $0.39,1.14$ & $0 \cdot 136$ \\
\hline \multicolumn{4}{|l|}{ Watch television/videos/listen to music daily } \\
\hline$\geq 2 \mathrm{~h} /<2 \mathrm{~h}$ & 1.89 & $1 \cdot 16,3.07$ & $0.011^{*}$ \\
\hline \multicolumn{4}{|l|}{ Read food labels } \\
\hline Sometimes and no/always and often & 1.50 & $0.92,2.45$ & $0 \cdot 107$ \\
\hline \multicolumn{4}{|l|}{ Arab girls $(n 726$, missing $=46)$} \\
\hline \multicolumn{4}{|l|}{ School level } \\
\hline High/middle school & $1 \cdot 30$ & $0.89,1.91$ & 0.179 \\
\hline \multicolumn{4}{|l|}{ Aerobic activity or ball games weekly } \\
\hline No/yes & 1.38 & $0.91,2.09$ & 0.135 \\
\hline \multicolumn{4}{|l|}{ Read food labels } \\
\hline Sometimes and no/always and often & 1.24 & $0.86,1.79$ & 0.256 \\
\hline
\end{tabular}

KIDMED, Mediterranean Diet Quality Index.

${ }^{\star} P<0.05,{ }^{\star \star} P<0.01$. 
(a)

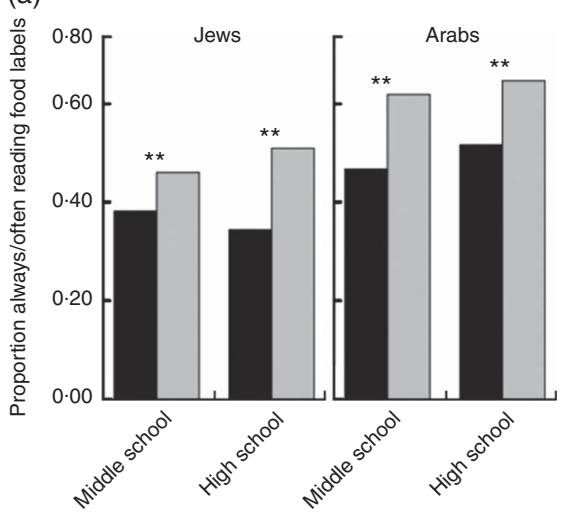

(b)

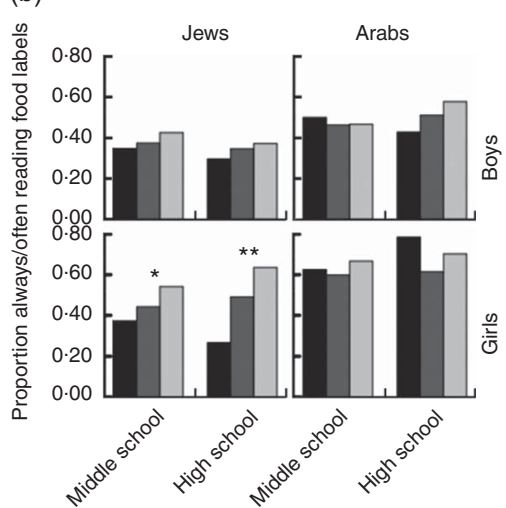

(c)

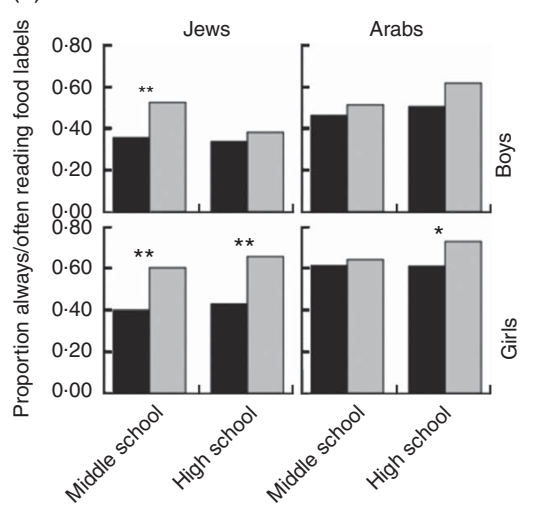

Fig. 1 The proportion of Israeli adolescents aged 11-19 years who always/often read food labels by gender, ethnicity, school level and selected factors; Mabat Youth Study (2003-2004). Always/often reading food labels was significantly associated with (a) gender ( $\square$, boys; $\square$, girls), (b) body weight ( $\square$, underweight; $\square$, normal weight; $\square$, overweight/obese) and (c) dieting ( $\square$, no dieting; $\square$, dieting): ${ }^{*} P<0.05,{ }^{\star \star} P<0.01$

always/often read food labels than Jewish children $(57.5 \mathrm{v}$. $43.2 \%, P<0 \cdot 001)$. When stratified by school level and ethnicity, the proportion of always/often reading food labels in girls was always significantly higher than that in boys (Fig. 1(a)).

When analysing the association between reading food labels and body weight, the proportion always/often reading food labels in the overweight/obesity group was almost always higher than that in the normal weight group, except in Arab boys in middle school (Fig. 1(b)). After stratification by gender, ethnicity and school level, the number of cases in the underweight category $(<5$ th percentile BMI) in some Arab groups was as small as less than ten. Therefore, the rate of reading food labels in underweight group in Arab children might not be reliable.

Dieting was also shown to be associated with frequency of reading food labels. Generally, adolescents who were dieting were more likely to always/often read food labels. The difference in the rate of always/often reading food labels between those who were and were not dieting was greater in Jews than in Arabs (Fig. 1(c)).

\section{KIDMED score and disordered eating}

When comparing the fourteen-item KIDMED scores between disordered eating (+) and disordered eating (-) groups, no difference in KIDMED scores were observed between the two groups when stratified by gender and ethnicity (data not shown).

\section{Discussion}

The present paper is a first attempt to describe the adherence to the MD using a modified KIDMED index in Israeli adolescents based on a nationwide health and nutrition survey - the Mabat Youth Survey. It has described the dietary patterns in Israeli adolescents, identified the high-risk group in terms of poor quality of eating and eating habits, and provided the direction for intervention programmes.

\section{KIDMED distribution}

The compliance to the MD was not very satisfactory in Israeli adolescents when compared with other youth populations of comparable age around the Mediterranean basin region. The overall distribution of fourteen-item KIDMED scores in the present study was that $25.5 \%$ of the total participants had poor, $55.2 \%$ had average and $19 \cdot 3 \%$ had good adherence to the MD. In other populations, Spanish children and adolescents seemed to have the best adherence to the MD, with only $2-7 \%$ having a poor and $30-50 \%$ having a good KIDMED score, according to different studies ${ }^{(11,16,26)}$. Surveys in other countries, including Greece $^{(19)}$, Turkey ${ }^{(23)}$ and the Balearic Islands ${ }^{(27)}$, demonstrated that the percentage of poor adherence to the MD at comparable ages ranged from 15 to $27 \%$, while the proportion of good compliance to the MD was between 8 and $28 \%$. In spite of the difference in cut-off values of KIDMED categories due to two missing items in the present study, the high proportion of poor adherence to the MD in Israeli adolescents was still alarming. It should be noted that the adolescents who completed the FFQ and thus were included in the KIDMED study represented 87.7 and $76 \cdot 1 \%$ of the Jewish and Arab participants in the Mabat Youth Survey, respectively. Further analysis showed that the noncompleters tended to be in middle school, with low SES and did not engage in physical activity regularly. Therefore, the true adherence to the MD in Israeli adolescents may be even less than the present data have shown. Geographically, Israel is in the Mediterranean basin; however, the diversity of the population in terms of ethnicity, religion, origin of immigration, family structure and more, may lead to a weaker tradition in compliance to the MD, which may explain the poor adherence to the MD in the present study. 
Further, looking at the KIDMED score distribution by school level, gender and ethnicity, Jewish middle-school children were identified as a high-risk group for low adherence to the MD, with an overall rate of having a poor KIDMED score of $28.2 \%$ (similar in boys and girls, Table 3 ). This was mainly attributable to less fruit and vegetable consumption, and more skipping breakfast (Table 2). Therefore, intervention programmes aimed at reversing these trends should be developed to improve the dietary quality and eating habits of this high-risk group.

\section{Factors associated with KIDMED score}

School level was inconsistently associated with KIDMED score between Jews and Arabs. Arab high-school children had poorer adherence to the MD than Arab middle-school children in terms of both the proportion of having a poor KIDMED score (Table 3 ) and the mean of KIDMED scores (Table 4). The trend disappeared in Jewish girls, and even reversed in Jewish boys (Tables 3 and 4). In other studies, the association between compliance to the MD and age, which was represented by school level in our study, was not consistent in different populations. A negative association between age and KIDMED score was found in most studies, including among Greek ${ }^{(19,21,22)}$, Spanish $^{(16)}$ and Balearic Islands ${ }^{(27)}$ schoolchildren. In fewer studies, KIDMED score did not differ among age groups ${ }^{(20,24)}$, although the age groups were not exactly the same as those in the present study. In addition to the effect of age, school education may also play a role in the trend of adherence to the MD by school level.

Mother's education was positively associated with better adherence to the MD in all groups of our study population (Table 4). In Jewish girls, the mother having less than 12 years of school education was the strongest explanatory factor for having a poor KIDMED score (Table 5). Similar findings were also observed in Spanish ${ }^{(11)}$, Greek $^{(19)}$, Balearic Islands ${ }^{(27)}$ and Turkish ${ }^{(23)}$ adolescents, highlighting the influential impact of mothers' education on their children, which should also be a focus for intervention.

Physical activity and sedentary lifestyle, as expected, showed opposite associations with the KIDMED scores. Performing aerobic activity or ball games weekly, and watching television/videos or listening to music for $<2 \mathrm{~h} / \mathrm{d}$ were consistently associated with better adherence to the MD (Tables 4 and 5). Such associations between physical activity, sedentary lifestyle and the KIDMED score have also been suggested in other studies ${ }^{(22,28,29)}$. In a study on adolescents in Tuscany, with a sample size of 1127 , the odds ratio for having a poor KIDMED score increased monotonically when frequency of physical activity decreased or time spent on sedentary activity increased, showing the consistency of the association ${ }^{(28)}$.

Since SES was classified based on the location of schools, without assessment at the individual level, the analysis by SES might be not reliable. This might explain the finding that, in Jewish girls, high SES was associated with poor adherence to the MD.

\section{Reading food labels and KIDMED score}

Always/often reading food labels was positively associated with better adherence to the MD in all study groups after stratification by gender and ethnicity (Tables 4 and 5). However, previous studies showed that reading food labels did not always translate into healthy food choices directly $^{(30)}$. One possible reason for our positive finding may be the facilitating role of nutrition knowledge in the association between reading food labels and better dietary quality. Evidence has shown that people who have better nutrition knowledge can utilize food label information better and make healthier food choices ${ }^{(30,31)}$. Another possible explanation is that the sub-populations who may be more conscious about their body weight, such as female students, heavier students and those who were dieting, may be more careful about their diets and therefore more likely to read food labels. In the present study, our data support the claim that these sub-populations do indeed read food labels more frequently (Fig. 1), but do not necessarily have higher KIDMED scores (Table 4). Because of the cross-sectional study design, the temporality of reading food labels and adherence to the MD was not known. Regardless of the pathway, for the positive association between reading food labels and better adherence to the MD, from the public health policy point of view, some facilitating factors should be considered to transform food label reading into healthy dietary choices and practices, such as providing key points of nutritional knowledge on the package, promoting reader-friendly food labels and advertising, etc. ${ }^{(30)}$. Also, psychological and social factors that affect eating behaviours in adolescence should also be considered.

The proportion of reading food labels among the Arab students was higher than that in the Jewish ones (Fig. 1(a)). This result was counter-intuitive, because Israeli law requires labelling in Hebrew only, which may have created some barriers in understanding food labels. A possible reason for the positive association was the differential selection bias between Jews and Arabs in the current KIDMED study. Among the national representative samples in the Mabat Youth Survey, the proportion of Jews and Arabs who completed the FFQ, thus being enrolled in the KIDMED study, was 87.7 and $76 \cdot 1 \%$, respectively. The non-completers had a significantly higher percentage of being in middle school or from a low-SES background, who would be less likely to read food labels frequently. Another possible reason was a social desirability bias ${ }^{(32)}$. Since the questionnaires were self-administered, participants may tend to give the expected answers to various extents depending on demographic, psychological and other factors. Because of the possible selection bias (rate difference in completing the FFQ between Jewish and Arab students) and the 
response bias (social desirability bias), the direct inter-ethnic comparisons might be not valid.

\section{Overweight/obesity and KIDMED score}

Overweight/obesity was not associated with adherence to the MD in the present study (Tables 4 and 5), which was consistent with studies in Greece ${ }^{(20,22)}$. In contrast, some studies have shown that overweight/obesity was associated with a higher KIDMED score ${ }^{(17,28)}$, whereas another study found a negative association between obesity and the KIDMED score ${ }^{(15)}$. These contradictory results demonstrate the complexity of this question. One explanation is the mediating effect of parents' educational status: a study in Greece showed that adherence to the MD in adolescents was inversely associated with children's obesity status only in families where parents had a higher educational level ${ }^{(33)}$. On the other hand, these inconsistencies might reflect certain drawbacks of the KIDMED index, which focuses only on dietary quality and healthy eating behaviours without considering the quantities of food consumed, and is therefore limited in its ability to predict overweight/obesity-related health outcomes.

\section{Study limitations}

There are several limitations to the present research. The main one was that the Mabat Youth Survey in general, and the FFQ in particular, was not specifically developed for the purpose of calculating a KIDMED score. Therefore, there were some necessary compromises in translating the data generated from the Mabat Youth Survey questionnaires to the KIDMED score, which could affect the comparability of our results with other KIDMED studies. However, we think that using fourteen of the sixteen questions with appropriate scaling still gave meaningful results. Second, the rate of completing the FFQ and thus being included in the current KIDMED study among the participants in Mabat Youth Survey was significantly higher in Jews than in Arabs (87.7 v. $76 \cdot 1 \%)$. This differential selection bias may influence the inter-ethnic comparisons. Third, the Mabat Youth Survey questionnaires were self-administered, which may cause the respondents to over-report socially desirable responses (social desirability response bias). Fourth, the SCOFF questionnaire was validated extensively in females, particularly in females more than 18 years old, but the validity test in boys was limited. Lastly, the data presented here relate to the year of 2004. The new Mabat Youth Survey, carried out in 20152016, will provide a new data set. Then the methodology discussed herein can be used and will allow following of time trends, which it is hoped will show an improvement in adherence to the MD.

\section{Conclusions}

In general, Israeli adolescents had poor adherence to the MD, especially among Jewish middle-school students.
Interventions aimed at increasing physical activity, reducing sedentary time, improving mother's education, promoting and facilitating reading of food labels are suggested, in order to improve nutritional status and promote the health of schoolchildren. Further research on the effectiveness of interventions targeting different factors in each subpopulation might be needed. The time trend of dietary pattern transition in adolescents also should be investigated.

\section{Acknowledgements}

Acknowledgements: This work was presented as part of the requirements of the International MPH programme in Braun School of Public Health, Hebrew University - Hadassah Medical School, Jerusalem. The authors are very grateful to Dr Mario Baras for kind statistical advice. They would also like to thank the Israel Center for Disease Control and the Ministry of Health, who conducted the survey together. Financial support: This research received no specific grant from any funding agency in the public, commercial or not-for-profit sectors. Conflict of interest: None. Authorship: All the authors participated in conceptualizing and designing the study; W.P. analysed the data and drafted the manuscript; R.G. and E.M.B. interpreted the data and revised the manuscript critically. Ethics of human subject participation: According to Ministry of Education guidelines, those students whose parents did not oppose, in writing, the participation of their children, were included in the survey, and given the self-administered questionnaire to complete.

\section{References}

1. Centers for Disease Control and Prevention (2012) Childhood Obesity Facts. http://www.cdc.gov/healthyschools/ obesity/facts.htm (accessed July 2016).

2. Nitzan Kaluski D, Demem Mazengia G, Shimony $\mathrm{T}$ et al. (2009) Prevalence and determinants of physical activity and lifestyle in relation to obesity among schoolchildren in Israel. Public Health Nutr 12, 774-782.

3. Vivante A, Golan E, Tzur D et al. (2012) Body mass index in 1.2 million adolescents and risk for end-stage renal disease. Arch Intern Med 172, 1644-1650.

4. Chen Y, Copeland WK, Vedanthan R et al. (2013) Association between body mass index and cardiovascular disease mortality in East Asians and South Asians: pooled analysis of prospective data from the Asia Cohort Consortium. BMJ 347, f5446.

5. Bjørge T, Engeland A, Tverdal A et al. (2008) Body mass index in adolescence in relation to cause-specific mortality: a follow-up of 230,000 Norwegian adolescents. Am J Epidemiol 168, 30-37.

6. Levi Z, Kark JD, Afek A et al. (2012) Measured body mass index in adolescence and the incidence of pancreatic cancer in a cohort of 720,000 Jewish men. Cancer Causes Control 23, 371-378.

7. Twig G, Yaniv G, Levine H et al. (2016) Body-mass index in 2.3 million adolescents and cardiovascular death in adulthood. $N$ Engl J Med 374, 2430-2440.

8. Serra-Majem L, Roman B \& Estruch R (2006) Scientific evidence of interventions using the Mediterranean diet: a systematic review. Nutr Rev 64,2 Pt 2, S27-S47. 
9. Sofi F, Abbate R, Gensini GF et al. (2010) Accruing evidence on benefits of adherence to the Mediterranean diet on health: an updated systematic review and meta-analysis. Am J Clin Nutr 92, 1189-1196.

10. United Nations Educational, Scientific and Cultural Organization (2013) Representative List of the Intangible Cultural Heritage of Humanity. http://www.unesco.org/culture/ich/ en/RL/mediterranean-diet-00884 (accessed April 2016).

11. Serra-Majem L, Ribas L, Ngo J et al. (2004) Food, youth and the Mediterranean diet in Spain. Development of KIDMED, Mediterranean Diet Quality Index in children and adolescents. Public Health Nutr 7, 931-935.

12. Grimm ER \& Steinle NI (2011) Genetics of eating behavior: established and emerging concepts. Nutr Rev 69, 52-60.

13. Gonzalez A, Kohn MR \& Clarke SD (2007) Eating disorders in adolescents. Aust Fam Physician 36, 614-619.

14. Kaluski DN, Natamba BK, Goldsmith R et al. (2008) Determinants of disordered eating behaviors among Israeli adolescent girls. Eat Disord 16, 146-159.

15. Schröder H, Mendez MA, Ribas-Barba L et al. (2010) Mediterranean diet and waist circumference in a representative national sample of young Spaniards. Int J Pediatr Obes 5, 516-519.

16. Mariscal-Arcas M, Rivas A, Velasco J et al. (2009) Evaluation of the Mediterranean Diet Quality Index (KIDMED) in children and adolescents in Southern Spain. Public Health Nutr 12, 1408-1412.

17. Grosso G, Marventano S, Buscemi S et al. (2013) Factors associated with adherence to the Mediterranean diet among adolescents living in Sicily, southern Italy. Nutrients 5, 4908-4923.

18. Roccaldo R, Censi L, D'Addezio L et al. (2014) Adherence to the Mediterranean diet in Italian school children (The ZOOM8 Study). Int J Food Sci Nutr 65, 621-628.

19. Kontogianni MD, Vidra N, Farmaki AE et al. (2008) Adherence rates to the Mediterranean diet are low in a representative sample of Greek children and adolescents. J Nutr 138, 1951-1956.

20. Farajian P, Risvas G, Karasouli K et al. (2011) Very high childhood obesity prevalence and low adherence rates to the Mediterranean diet in Greek children: the GRECO study. Atherosclerosis 217, 525-530.

21. Bargiota A, Pelekanou M, Tsitouras A et al. (2013) Eating habits and factors affecting food choice of adolescents living in rural areas. Hormones (Athens) 12, 246-253.
22. Papadaki S \& Mavrikaki E (2015) Greek adolescents and the Mediterranean diet: factors affecting quality and adherence. Nutrition 31, 345-349.

23. Sahingoz SA \& Sanlier N (2011) Compliance with Mediterranean Diet Quality Index (KIDMED) and nutrition knowledge levels in adolescents. A case study from Turkey. Appetite 57, 272-277.

24. Lazarou C, Panagiotakos DB \& Matalas AL (2009) Level of adherence to the Mediterranean diet among children from Cyprus: the CYKIDS study. Public Health Nutr 12, 991-1000.

25. Luck AJ, Morgan JF, Reid F et al. (2002) The SCOFF questionnaire and clinical interview for eating disorders in general practice: comparative study. BMJ 325, 755-756.

26. Grao-Cruces A, Nuviala A, Fernández-Martínez A et al. (2013) Adherence to the Mediterranean diet in rural and urban adolescents of southern Spain, life satisfaction, anthropometry, and physical and sedentary activities. Nutr Hosp 28, 1129-1135.

27. Bibiloni Mdel M, Pons A \& Tur JA (2016) Compliance with the Mediterranean Diet Quality Index (KIDMED) among Balearic Islands' adolescents and its association with socioeconomic, anthropometric and lifestyle factors. Ann Nutr Metab 68, 42-50.

28. Santomauro F, Lorini C, Tanini T et al. (2014) Adherence to Mediterranean diet in a sample of Tuscan adolescents. Nutrition 30, 1379-1383.

29. Arriscado D, Muros JJ, Zabala M et al. (2014) Factors associated with low adherence to a Mediterranean diet in healthy children in northern Spain. Appetite 80, 28-34.

30. Auchincloss AH, Young C, Davis AL et al. (2013) Barriers and facilitators of consumer use of nutrition labels at sit-down restaurant chains. Public Health Nutr 16, $2138-2145$.

31. Miller LMS \& Cassady DL (2015) The effects of nutrition knowledge on food label use. A review of the literature. Appetite 92, 207-216.

32. Fisher RJ \& Katz JE (2000) Social-desirability bias and the validity of self-reported values. Psychol Mark 17, 105-120.

33. Antonogeorgos G, Panagiotakos DB, Grigoropoulou D et al. (2013) The mediating effect of parents' educational status on the association between adherence to the Mediterranean diet and childhood obesity: the PANACEA study. Int J Public Health 58, 401-408. 